\title{
Urinary Tract Resections in Advanced-stage Cervical Cancer - A Series of Eight Cases
}

\author{
DANA NUTI OPRESCU ${ }^{1}$, NICOLAE BACALBASA ${ }^{2}$, IRINA BALESCU $^{3}$ and ALEXANDRU FILIPESCU ${ }^{4}$ \\ ${ }^{1}$ Department of Obstetrics and Gynecology, INSMC “Alessandrescu Rusescu”, Bucharest, Romania; \\ ${ }^{2}$ Department of Obstetrics and Gynecology, Carol Davila University of Medicine and Pharmacy, Bucharest, Romania; \\ ${ }^{3}$ Department of General Surgery, Ponderas Academic Hospital, Bucharest, Romania; \\ ${ }^{4}$ Department of Obstetrics and Gynecology, Elias Hospital, Bucharest, Romania
}

\begin{abstract}
Background/Aim: Cervical cancer is one of the most frequent malignancies in women worldwide and is unfortunately diagnosed in advanced stages of the disease. Whenever local invasion is present, neoadjuvant therapy might be needed in order to limit the degree of local invasion. However, in certain cases local invasion persists even after completing the neoadjuvant radiochemotherapy; in these patients more extensive resections might be needed in order to achieve a radical resection. Patients and Methods: We present a case series of eight patients in whom segmental ureteral or uretero-vesical resections were performed as part of the radical resections for locally advanced cervical tumors. Results: The continuity of the urinary tract was re-established by performing ureteral reimplantation via uretero-neocystostomy, augmentation cystoplasties with ureteral reimplantations. In a single case ureteral reimplantation was not feasible, a definitive cutaneous ureterostomy being performed. The postoperative course was uneventful in seven cases while in a single case urinary leak occurred, necessitating the exteriorization of the ureter in terminal cutaneous ureterostomy. Conclusion: Ureteral resections can be safely performed in patients with locally advanced cervical cancer.
\end{abstract}

Advanced cervical cancer is defined by the presence of a maximum tumoral diameter of at least $4 \mathrm{~cm}$, FIGO (The International Federation of Gynecology and Obstetrics) stage $>$ IIA or relapsed tumors and has been advocated to represent up to $50 \%$ of cases, especially in developing countries $(1,2)$. When it comes to the standard therapeutic options in cervical cancer, cases diagnosed in FIGO stages I and II can be submitted to radical hysterectomy with pelvic and eventually

Correspondence to: Irina Balescu, Caraiman Street, no. 2A, Buzau, Romania. Tel: +40 724077709, e-mail: irina_balescu206@yahoo.com

Key Words: Locally advanced cervical cancer, ureteral resection, urinary reconstruction. para-aortic lymph node dissection as the first therapeutic option; in the meantime, patients diagnosed in advanced stages of the disease (FIGO stage III and IVA) are treated with chemoradiation excepting the cases in which radiation therapy is hampered (by the presence of vesicovaginal or rectovaginal fistulas). However, in certain cases, although neoadjuvant chemotherapy is performed local invasion of the surrounding viscera persists; in all these cases multiple visceral resections might be needed in order to achieve a good local control of the disease. Due to the close proximity of the urinary bladder and of the rectum with the uterine cervix, these segments are most often affected by the neoplastic process. Depending on the degree of invasion, different variants of resections have been proposed; while cases presenting a high grade of local invasion are usually treated by performing a pelvic exenteration, cases with a lower degree of invasion are suitable to a more conservative type of surgery consisting of segmental digestive or urinary resections followed by anastomosis of the remnant partners (3).

\section{Materials and methods}

Between 2015 and 2016 eight patients with advanced-stage cervical cancer and persistent invasion of the urinary tract were submitted to radical surgery followed by urinary tract reconstruction. In all cases ureteral reimplantations were protected by placing ureteral stents which were removed 21 days postoperatively. Postoperative complications were classified according to Dindo-Clavien scale (4).

\section{Results}

All eight cases were initially diagnosed with locally advanced cervical cancer with urinary tract involvement, so they were initially submitted to neo-adjuvant chemoirradiation. Seven of the eight cases successfully performed the entire neoadjuvant protocol while in the eight case the radiation therapy was interrupted due to the apparition of a severe hemorrhagic episode, so the patient was submitted to surgery in emergency. The main characteristics of the 
Table I. The main intraoperative characteristics of the group of eight patients.

\begin{tabular}{|c|c|c|c|c|}
\hline & $\begin{array}{l}\text { Age } \\
\text { (years) }\end{array}$ & $\begin{array}{l}\text { Localization of the } \\
\text { invaded segment }\end{array}$ & Type of resection & Urinary tract reconstruction \\
\hline Case 1 & 57 & Left ureteral ostium & Distal ureterectomy & Ureteral reimplantation through neo-cystostomy \\
\hline Case 2 & 53 & Bilateral ureteral ostium & $\begin{array}{l}\text { Partial cystectomy, } \\
\text { bilateral ureteral resection }\end{array}$ & Bilateral reimplantation through neo-cystostomy \\
\hline Case 3 & 48 & $\begin{array}{l}\text { Bilateral ureteral ostium, } \\
\text { bladder trigone }\end{array}$ & $\begin{array}{l}\text { Partial cystectomy, } \\
\text { bilateral ureteral resection }\end{array}$ & Bilateral reimplantation through neo-cystostomy \\
\hline Case 4 & 42 & Left ureteral ostium & Distal ureterectomy & Ureteral reimplantation through neo-cystostomy \\
\hline Case 5 & 44 & Right ureteral ostium & Distal ureterectomy & Ureteral reimplantation through neo-cystostomy \\
\hline Case 6 & 59 & $\begin{array}{l}\text { Bilateral ureteral ostium, } \\
\text { bladder trigone }\end{array}$ & $\begin{array}{l}\text { Partial cystectomy en bloc with } \\
\text { distal ureterectomy }\end{array}$ & $\begin{array}{l}\text { Augmentation cystoplasty, ureteral } \\
\text { reimplantation through neo-cystostomy }\end{array}$ \\
\hline Case 7 & 62 & Left ureteral ostium & Distal ureterectomy & Cutaneous ureterostomy \\
\hline Case 8 & 51 & Right ureteral ostium & Distal ureterectomy & Ureteral reimplantation through neo-cystostomy \\
\hline
\end{tabular}

patients are presented in Table I. The main intraoperative aspects are shown in Figures 1-10.

In all cases ureteral reimplantation through neocystostomy was attempted; however, in a case due to the presence of a high grade of retroperitoneal fibrosis affecting more than a half of the ureter, we decided to create a cutaneous ureterostomy. All the reimplantation procedures were protected by placing Cook ureteral catheters which were removed through cystoscopy at the end of the first month postoperatively (days 21-30). The postoperative course was uneventful in seven cases, while in a single case a 4 grade Dindo-Clavien complication occurred; the patient developed a postoperative febrile episode and a urinary leak was diagnosed in the seventh postoperative day. The patient had been submitted to surgery for locally advanced cervical cancer with invasion into the bladder trigon; although initially both ureters were reinserted in the remnant urinary bladder, the apparition of the urinary leak imposed reoperation and exteriorization of the both ureters in terminal cutaneous ureterostomy. The mean length of stay in hospital was 7 days (range 6-38 days).

\section{Discussion}

Cervical cancer is part of the solid types of tumors which are characterized by progressive tissue destruction, a process known under the generic name of morpholysis. Throughout this process the malignant disease provides an isotopic invasion of the tumor cells into the peritumoral tissues (5). Although initially this process is limited by the compartimental natural borders, defined by the embryological development of the pelvic viscera, these borders seem to be destroyed by the evolution of the malignant process. It has been postulated that in the early stages of the disease the malignant solid tumors spread on the paths of the least mechanical resistance, defined by the anatomical landmarks of the morphogenetic unit of the viscera from which the tumor originates (6). It has been demonstrated that in women the genital ducts (consisting of fallopian tubes, corpus and cervix uteri as well as the vagina) originate from the Mullerian ducts except from the distal part of the vagina which originates in the Wolffian ducts (6). During the embriogenetic process three distinct morphogenetic units rise: the proximal, intermediate and distal units. The proximal units consist of the fallopian tubes and the mesosalpinx, the intermediate unit consists of the uterine corpus and the broad ligaments while the distal unit consists of the uterine cervix, vagina, the neurovascular structures and the connective tissue, has a subperitoneal disposition and is the most complex segment (7). Studies have shown that in cases presenting with low stages of disease (FIGO stage IB-IIB), the malignant process is confined to the Mullerian morphogenetic unit; due to this aspect, according to Hockel's study, in these cases total mesometrial resection provides an R0 resection and minimizes the rates of local failure even in the absence of adjuvant radiotherapy (6). In this way, in cases in which the compartimental borders are not destroyed, the resection of the tumor en bloc with the tumorpermissive domain allows the preservation of the other structures situated in the close proximity to the malignant process and minimizes the surgery related morbidity (8). This type of surgical approach was defined by Michael Hockel as radical compartimentalized surgery and refers to the removal of a morphogenetic unit or a part of it.

During a slowly developing tumor, when the natural borders are destroyed, malignant cells can invade the surrounding viscera and compartments; in all these cases more aggressive surgical procedures might be needed in order to achieve a good control of the disease. In this way the concept of ultra-radical compartmentalized surgery developed; this concept refers to the en bloc removal of the 


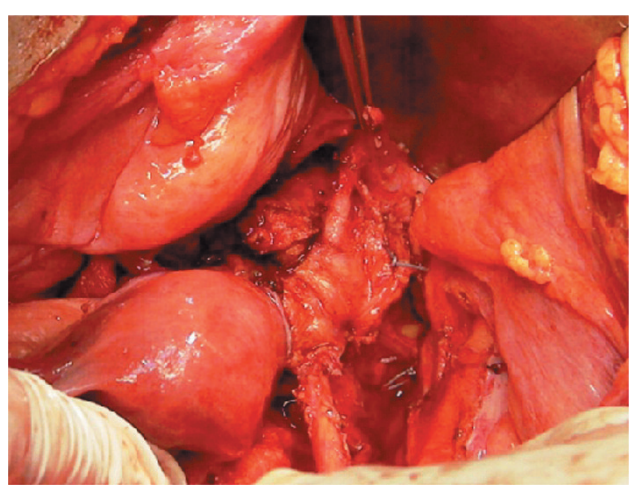

Figure 1. Locally advanced cervical cancer invading the right ureter.

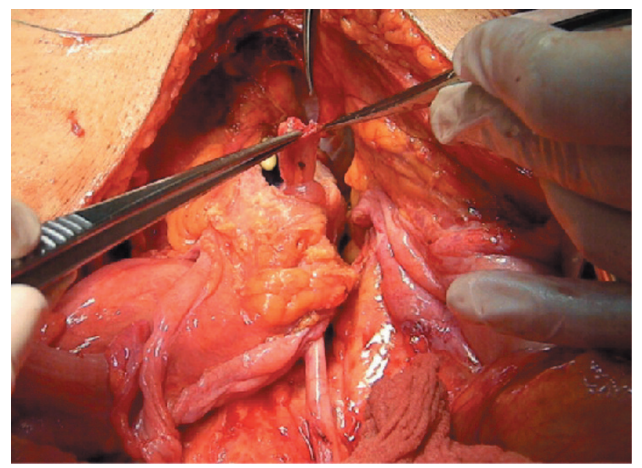

Figure 2. Right ureter reimplantation through uretero-neocsytostomy.

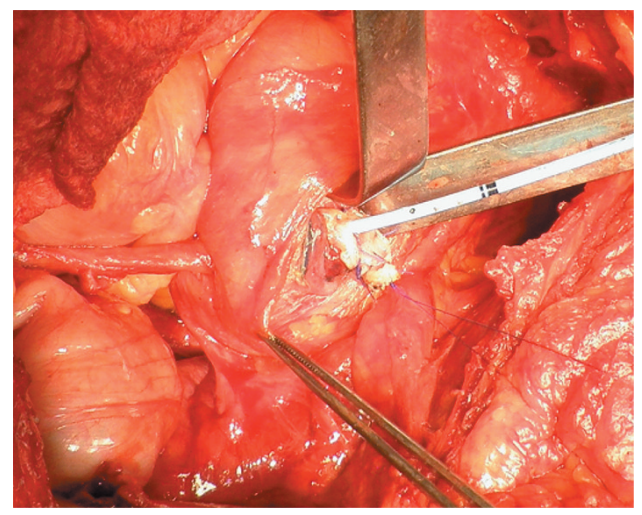

Figure 3. Placing the ureteral stent.

tumor en bloc with the invaded pelvic viscera, the cornerstone procedure being pelvic exenteration $(9,10)$. However, in gynecologic oncology patients, in certain cases in which the

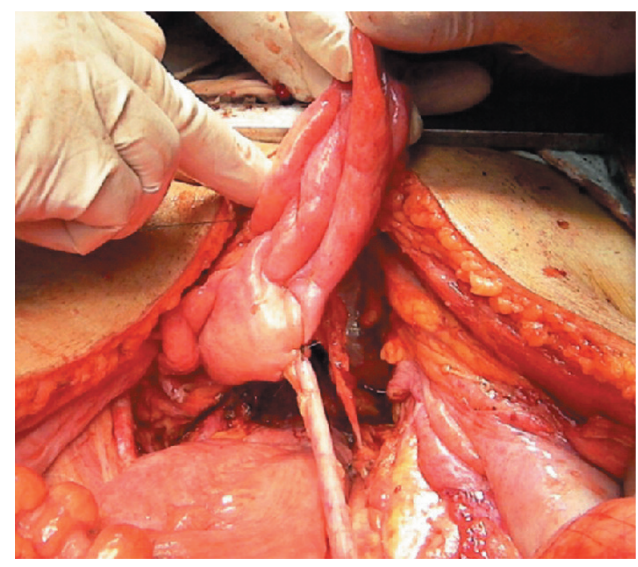

Figure 4. The final aspect after ureteral reimplantation and mobilization of the urinary bladder.

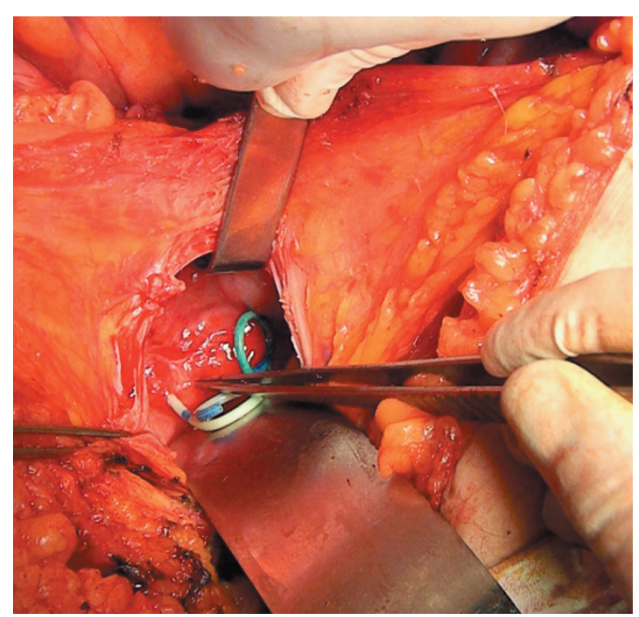

Figure 5. Cervical tumor with bladder trigon invasion. The two ureters were stented preoperatively with double J catheters.

local invasion is not so important, more conservative procedures might be taken in consideration (11-13).

In the study conducted by Mitchel Hoffmanin 2006 regarding the necessity of performing ureteral surgery in university gynecologic oncology service, the authors included 4,844 patients that were submitted to surgery between 1997 and 2004 for gynecologic malignancies. Among these cases ureteral surgery was performed in 46 cases, 16 patients being submitted to ureteral resection due to the extent of the malignant process. The indication for ureteral resection consisted of locally advanced or relapsed ovarian cancer (in nine cases), vaginal cancer (in two cases), recurrent cervical cancer (in two cases), class $\mathrm{V}$ radical hysterectomy (consisting in en bloc removal of the distal ureter and partial cystectomy 


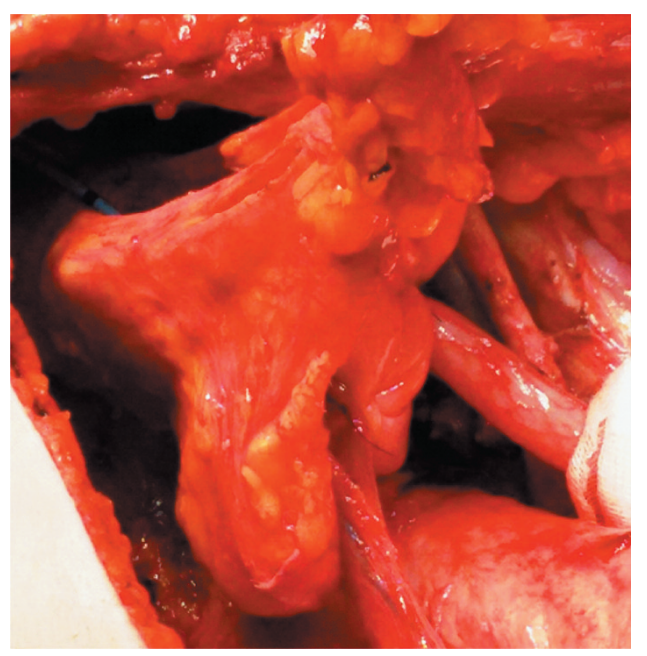

Figure 6. The two ureters are reimplanted through bilateral ureteoneocystostomy.

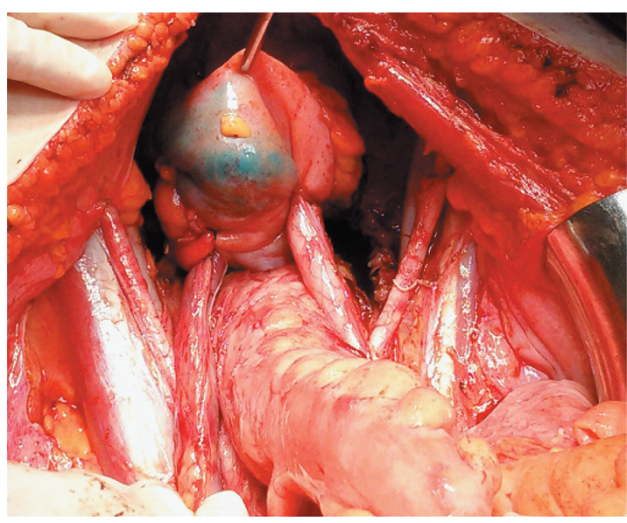

Figure 7. The final aspect after bilateral ureteral reimplantation.

for locally advanced tumors - in two cases) and bilateral intrinsic ureteral endometriosis in one case. The authors also reported that partial cystectomies were associated in three cases while the mean upper level of ureteral transection was estimated to be $5.7 \mathrm{~cm}$ (range $2-10 \mathrm{~cm}$ ). In all cases the continuity of the urinary tract was re-established through uretero-neocystostomy. Moreover, in one case presenting bilateral ureteral invasion both ureters were re-inserted in the remnant urinary bladder through uretero-neocystostomy. Regarding the postoperative outcome, a single patient developed a major complication requiring re-operation (a vesicovaginal fistula which required reoperation at two weeks postoperatively); this complication was treated by performing an ileal conduit. Other less severe complications consisted of

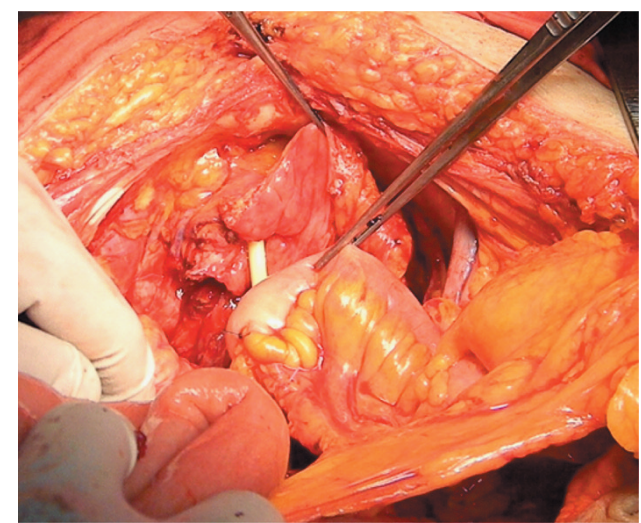

Figure 8. Augmentation cystoplasty using an ileal loop - preparing the anastomosis.

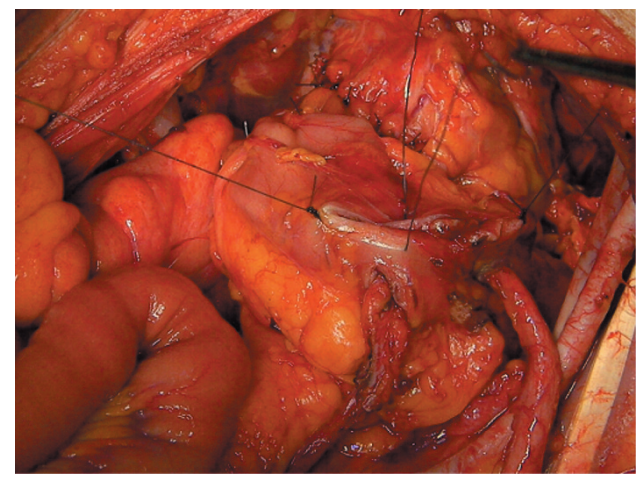

Figure 9. Insertion of the two ureters in the neo-bladder.

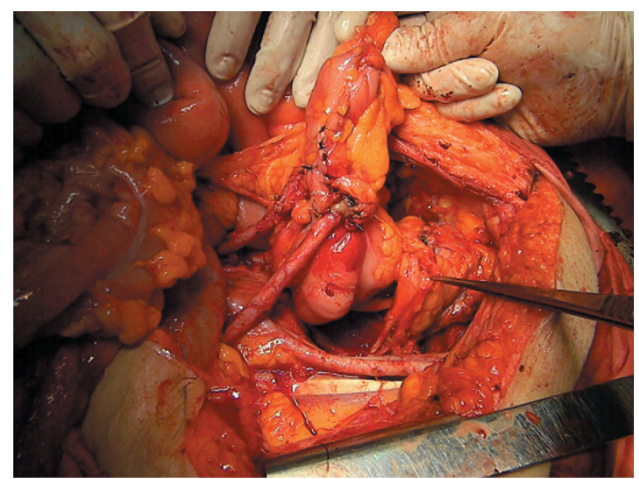

Figure 10. The final aspect after augmentation cystoplasty and bilateral ureteral reimplantation.

the persistence of the aspect of a hydroureter after removing the ureteral stent or the apparition of urinary incontinence due to the small capacity of the urinary bladder (12). 
Regarding the surgical technique of the ureteral neocystostomy, two options have been reported so far: with or without tunnel anastomosis. When performed in children (as an anti-reflux procedure) or in young women (who are submitted to conservative fertility sparing procedures and who plan to obtain a pregnancy), a tunneled anastomosis should be the option of choice $(14,15)$. In all the other cases, the surgeon can chose between the two options in function of the anatomical particularity of each case. In our study all cases were submitted to a non-tunneled anastomosis. In order to achieve a tension-free anastomosis, various technical tips such as renal or bladder mobilization were used.

However, when it comes to urinary tract reconstruction after ureteral resection for locally invasive cervical tumors, other surgical techniques such as ileal ureter, transureteroureterostomy or cutaneous ureterostomy might be taken into consideration according to the anatomical particularities of each patient $(12,16)$.

When it comes to the role of augmentation cystoplasty in re-establishing the continuity of the urinary tract in patients with a more extended area of tumoral invasion at the level of the urinary bladder, this method was performed in the current study in a single patient who presented a cervical tumor invading both ureteral ostiums as well as the bladder trigon. After resection the remnant urinary ,bladder volume was insufficient, so we decided to perform an augmentation cystoplasty using an ileal loop, followed by bilateral ureteral reinsertion. The method was initially used in children with vesico-ureteral reflux as well as in cases with neurogenic bladder. This technique has multiple advantages: it increases the urinary bladder volume, it decreases intravesical pressure and, in the meantime improves the compliance (17-19). Other digestive segments which have been used for augmentation cystoplasty include jejunal loops or colonic segments. Although most authors consider that small bowel segments are even more appropriate in order to enlarge the remnant urinary bladder (due to the rich blood supply and due to the capacity of providing an extremely low pressure system) (20), attention should be given to certain aspects: ileal segments might induce severe metabolic dysfunctions such as hyperchloremic metabolic acidosis and might also have an increased risk of malignant transformation of the epithelium $(21,22)$.

\section{Conclusion}

Ureteral resections can be safely performed as part of the ultra-radical surgical procedures for locally advanced cervical cancer in order to achieve negative resection margins and a good control of the disease, with acceptable rates of postoperative complications. As for the most appropriate technique, different procedures have been proposed, depending on the extent of the resected area.

\section{Acknowledgements}

This work was supported by a grant of the Romanian National Authority for Scientific Research and Innovation, CNCS UEFISCDI, project number PN-II-RU-TE-2014-4-2533.

\section{References}

1 Benedet JL, Odicino F, Maisonneuve P, Beller U, Creasman WT, Heintz AP, Ngan HY, Sideri M and Pecorelli S: Carcinoma of the cervix uteri. J Epidemiol Biostat 6: 7-43, 2001.

2 Ferlay J, Bray F, Pisani P and Parkin D Globocan, 2000. Cancer incidence, mortality and prevalence worldwide. Lyon: International Agency for Research on Cancer, WHO, IARC Press, 2001.

3 Hockel $\mathrm{M}$ and Dornhofer N: How to manage locally advanced primary and recurrent cancer of the uterine cervix: The surgeon's view. Reviews in Gynaecological Practice 5: 212-220, 2005.

4 Dindo D, Demartines $\mathrm{N}$ and Clavien PA: Classification of surgical complications: a new proposal with evaluation in a cohort of 6336 patients and results of a survey. Ann Surg 240: 205-213, 2004.

5 Hockel M, Hentschel B and Horn LC: Association between developmental steps in the organogenesis of the uterine cervix and locoregional progression of cervical cancer: a prospective clinicopathological analysis. Lancet Oncol 15: 445-456, 2014.

6 Hockel M, Horn LC and Fritsch H: Association between the mesenchymal compartment of uterovaginal organogenesis and local tumour spread in stage IB-IIB cervical carcinoma: a prospective study. Lancet Oncol 6: 751-756, 2005.

7 Fritsch H: The connective tissue sheath of uterus and vagina in the human female fetus. Ann Anat 174: 261-266, 1992.

8 Hockel M: Morphogenetic fields of embryonic development in locoregional cancer spread. Lancet Oncol 16: e148-e151, 2015.

9 Hockel M: Ultra-radical compartmentalized surgery in gynaecological oncology. Eur J Surg Oncol 32: 859-865, 2006.

10 Brunschwig A: Complete excision of pelvic viscera for advanced carcinoma; a one-stage abdominoperineal operation with end colostomy and bilateral ureteral implantation into the colon above the colostomy. Cancer 1: 177-183, 1948.

11 Bacalbasa N, Balescu I and Tomescu D: Partial cystectomy with distal ureteral resection and re-implantation for locally invasive cervical cancer. Anticancer Res 35: 5539-5542, 2015.

12 Hoffman MS and Tebes SJ: Ureteral surgery performed by a university gynecologic oncology service. Am J Obstet Gynecol 195: 562-566, 2006.

13 Chiva LM, Lapuente F, Gonzalez-Cortijo L, Gonzalez-Martin A, Rojo A, Garcia JF and Carballo N: Surgical treatment of recurrent cervical cancer: state of the art and new achievements. Gynecol Oncol 110: S60-S66, 2008.

14 Mendez LE: Iatrogenic injuries in gynecologic cancer surgery. Surg Clin North Am 81: 897-923, 2001.

15 Mandal AK, Sharma SK, Vaidyanathan S and Goswami AK: Ureterovaginal fistula: summary of 18 years' experience. Br J Urol 65: 453-456, 1990.

16 Manolitsas TP, Copeland LJ, Cohn DE, Eaton LA and Fowler JM: Ureteroileoneocystostomy: the use of an ileal segment for ureteral substitution in gynecologic oncology. Gynecol Oncol 84: 110-114, 2002. 
17 Soylet Y, Emir H, Ilce Z, Yesildag E, Buyukunal SN and Danismend N: Quo vadis? Ureteric reimplantation or ignoring reflux during augmentation cystoplasty. BJU Int 94: 379-380, 2004.

18 Simforoosh N, Tabibi A, Basiri A, Noorbala MH, Danesh AD and Ijadi A: Is ureteral reimplantation necessary during augmentation cystoplasty in patients with neurogenic bladder and vesicoureteral reflux? J Urol 168: 1439-1441, 2002.

19 Wang JB, Liu CS, Tsai SL, Wei CF and Chin TW: Augmentation cystoplasty and simultaneous ureteral reimplantation reduce high-grade vesicoureteral reflux in children with neurogenic bladder. J Chin Med Assoc 74: 294-297, 2011.
20 Elkas JC, Berek JS, Leuchter R, Lagasse LD and Karlan BY: Lower urinary tract reconstruction with ileum in the treatment of gynecologic malignancies. Gynecol Oncol 97: 685-692, 2005.

21 Nurse DE and Mundy AR: Metabolic complications of cystoplasty. Br J Urol 63: 165-170, 1989.

22 Nurse DE and Mundy AR: Assessment of the malignant potential of cystoplasty. Br J Urol 64: 489-492, 1989.

Received April 16, 2017

Revised April 30, 2017

Accepted May 2, 2017 